\title{
PREVALÊNCIA E CUSTOS INDIRETOS DAS CEFALÉIAS EM UMA EMPRESA BRASILEIRA
}

\author{
MAURICE VINCENT*, ANDREIA DE JESUS RODRIGUES**, GISELE VIEIRA DE OLIVEIRA**, \\ KARINE FREITAS DE SOUZA**, LARISSA MORIMOTO DOI**, \\ MANOELA BITTENCOURT DE LIMA ROCHA**, MÁRIO ANDRÉ DA CUNHA SAPORTA**, \\ RICARDO BENEDETTO ORLEANS ${ }^{* *}$, ROSANA KOTECKI***, VANESSA VIEIRA ESTRELA**, \\ VIVIANE ANTOONIO DE MEDEIROS **, WANDER INTURIAS SERGILLO BORGES**
}

\begin{abstract}
RESUMO - Funcionários de uma empresa $(\mathrm{n}=993)$ foram entrevistados quanto à ocorrência de cefaléias durante um período retrospectivo de 30 dias. A prevalência foi $49,8 \%$, com frequência de 4,3 $\pm 7,0$ episódios e duração de $12,2 \pm 21,4$ horas. Os diagnósticos baseados na classificação da Sociedade Internacional de Cefaléias, foram enxaqueca (5,5\%), cefaléia do tipo tensão (CTT) episódica (26,4\%), CTT crônica $(1,7 \%)$ e outras cefaléias $(16,2 \%)$. As mulheres foram mais acometidas e tiveram proporcionalmente mais enxaquecas que os homens. Cerca de $10 \%$ dos pacientes relataram dor suficientemente intensa a ponto de prejudicar seu desempenho no trabalho, o que representou 538,75 horas não trabalhadas. O custo indireto proporcionado pela interferência no trabalho foi estimado para cada cefaléia. O potencial prejuízo projetado à empresa devido às cefaléias é $\mathrm{R} \$ 145,64$ por funcionário, ou $\mathrm{R} \$ 144682,39$ por ano. Como a enxaqueca é a cefaléia de maior custo, seu controle é particularmente importante no ambiente de trabalho. Há meios eficazes para reduzir sua frequência, com reflexos positivos no bem-estar e na produtividade do indivíduo. A relação custo-benefício favorece claramente a instituição de programas de prevenção e tratamento contra cefaléias crônicas.
\end{abstract}

PALAVRAS-CHAVE: cefaléia, enxaqueca, epidemiologia, custos.

\section{Prevalence and indirect costs of headache in a Brazilian Company}

ABSTRACT - Employees from a Brazilian oil company reserach centre ( $n=993)$ were interviewed on the occurrence of headache during a 30 days period. Headache prevalence was $49.8 \%$, with a mean frequency of $4.3 \pm 7.0$ attacks per month, lasting 12.2 \pm 21.4 hours each. According to the International Headache Society diagnostic criteria, migraine (5.5\%), episodic tension-type headache (26.4\%), chronic tension-type headache $(1.7 \%)$ and headaches not fulfilling the criteria for such disorders $(16.2 \%)$ were observed. Women suffered comparatively more headache and specifically migraine than men. The pain interfered with work productivity in $10 \%$ of the subjects, corresponding to 538.75 hours off. According to an indirect costs estimation for each headache, the company may loose up to US\$125.98 per employee annually. Since among headaches migraine has the highest indirect cost, migraine prevention and treatment is particularly important at the working environment. Migraine frequency may be prevented to a large extent, resulting on positive effects in both the quality of life and productivity. The cost-benefit ratio clearly favours therapeutic and preventive programs against chronic headaches.

KEY WORDS: headache, migraine, epidemiology, costs.

Poucos são os indivíduos que jamais experimentam um episódio de cefaléia ${ }^{1,2}$. Noventa por cento dos homens e $95 \%$ das mulheres admitem ter sofrido algum tipo de dor de cabeça durante 1

Setor de Cefaléias, Serviço de Neurologia, Hospital Universitário Clementino Fraga Filho; Faculdade de Medicina, Universidade Federal do Rio de Janeiro (UFRJ), Brazil: *Professor Adjunto; **Aluno de Graduação; ***Médica Residente. Aceite: 27-julho-1998.

Dr. Maurice Vincent - Av. das Américas 1155/504 - 22631-000 Rio de Janeiro RJ - Brasil. Fax: +55 214943648. E-mail: vincent@unisys.com.br 
$a^{3} o^{3}$. As cefaléias secundárias constituem sintomas de outras afecções. Ao contrário, nas cefaléias primárias, mais frequentes, não há uma causa subjacente ${ }^{4}$. As principais cefaléias primárias quanto à frequência são a enxaqueca (ou migrânea) e a cefaléia do tipo tensão (CTT). Isoladas ou combinadas, estas duas condições respondem pela maioria das dores de cabeça ${ }^{2,5}$.

As cefaléias, particularmente a enxaqueca, podem influenciar negativamente o bem-estar do indivíduo e determinar prejuízos para a sociedade ${ }^{6}$. Suas consequências envolvem custos diretos (gastos com o sistema de saúde) e indiretos (prejuízos pela falta ao trabalho e diminuição da produtividade $)^{7}$. Os custos indiretos podem representar de 76 a $86 \%$ do impacto provocado pela enxaqueca ${ }^{8}$. O prejuízo anual causado pela população trabalhadora enxaquecosa nos EUA foi avaliado em US $\$ 1,4$ a 17 bilhões ${ }^{7,9}$. Estima-se que os custos indiretos possam ser ainda maiores, pois não contabilizam os prejuízos no trabalho doméstico, não remunerado.

Neste estudo, funcionários da Petrobrás foram entrevistados quanto à ocorrência de cefaléias, suas características clínicas e ao potencial de interferência na produtividade. Este é o primeiro estudo sobre o impacto econômico das cefaléias no ambiente de trabalho realizado no Brasil segundo critérios da "International Headache Society" (IHS).

\section{MATERIAL E MÉTODOS}

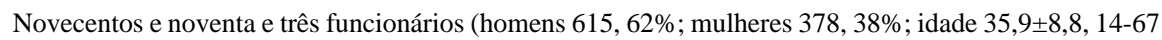
Fig 1a) do Centro de Pesquisas da Petrobrás (CENPES), Ilha do Fundão, Rio de Janeiro, foram entrevistados individualmente por estudantes da Faculdade de Medicina da UFRJ. Os 11 entrevistadores foram previamente treinados e familiarizados com o questionário (Tabela 1). Os funcionários foram orientados a informar a respeito da ocorrência de cefaléia apenas durante os 30 dias imediatamente anteriores à entrevista. As informações referentes à presença de náuseas, vômitos, foto e fonofobia foram subjetivamente divididas pelos entrevistados em ausente, leve, moderada e intensa.

Tabela 1. Questionário.

\begin{tabular}{ll}
\hline Pergunta & Resposta \\
\hline Identificação & Iniciais e matrícula do funcionário \\
Sexo & m: masculino; f: feminino \\
idade & anos \\
Teve cefaléia nos últimos 30 dias? & s: sim; n: não \\
Com que frequência tem cefaléias usualmente? & crises por mês \\
Duração da crise & horas \\
Localização da crise & d: direita; e: esquerda; b: bilateral \\
Caráter da dor & a: aperto, pressão; p: pulsátil, latejante \\
Intensidade da crise & 1: leve (não interfere com o trabalho); $2:$ moderada a \\
& intensa (interfere com o trabalho); $3:$ insuportável \\
& (impede o trabalho, leva ao leito) \\
Náuseas & n: não; l: leve; m: moderada; i: intensa* \\
Vômitos & n: não; l: leve; m: moderada; i: intensa* \\
Fotofobia & n: não; l: leve; m: moderada; i: intensa* \\
Fonofobia & n: não; l: leve; m: moderada; i: intensa* \\
Exercício físico leve & p: piora; m: melhora; na: não altera \\
Sintomas que antecedem a dor & descrição pelo entrevistado \\
Alteração visual antes da dor & descrição pelo entrevistado \\
\hline
\end{tabular}

*Definição subjetiva, feita pelo entrevistado. 


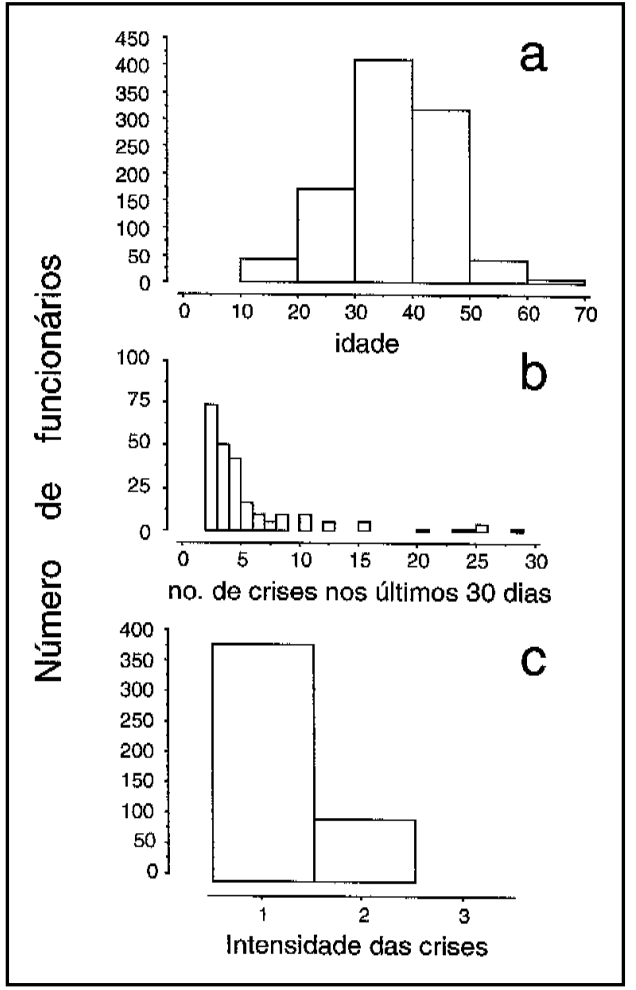

Fig 1. Distribuição dos funcionários de acordo com a faixa etária (a); frequência de crises de cefaléia, (b) número de crises (c). Intensidade 1: não interfere com o trabalho. Intensidade 2: Interfere com o trabalho. Intensidade 3: Insuportável, excruciante.

\section{sofrem em media $5,2 \pm 7,5(2$ de 0 a 30$)$ crises por mes. A d}

Os dados foram analisados de acordo com os critérios diagnósticos da IHS ${ }^{4}$, e as crises classificadas nas categorias enxaqueca, CTT (episódica ou crônica). e outra cefaléia (OC, não preenchendo critérios para enxaqueca nem CTT). A duração dos ataques em cada categoria e seu grau de interferência no trabalho foram utilizados para estimar os custos indiretos relacionados às cefaléias, considerando, para a empresa, uma despesa de $\mathrm{R} \$ 10,00$ (US\$8.65)/ hora / funcionário. A despesa com cada funcionário é superior ao salário. Consideramos também 176 horas de trabalho por mês, e 11 meses de trabalho por ano. Como o trabalho ocupa cerca de $1 / 4$ do tempo, de cada 4 horas com cefaléia 1 tem chances de ocorrer durante o expediente. Os valores em US\$ foram calculados de acordo com o câmbio comercial de 30/junho/1998.

Os dados são apresentados como média \pm desvio padrão (mediana, valor mínimo - valor máximo da amostra). Os testes Qui-quadrado, t de Student e ANOVA foram usados para comparações estatísticas. Valores de $\mathrm{p}<0.05$ foram considerados significativos.

\section{RESULTADOS}

Metade dos entrevistados (495-49,8\%) revelou ter sofrido cefaléia nos últimos 30 dias, em média 4,3 7,0 (2 de 1 a 30) episódios, na sua maioria poucas crises (Fig 1b), durando 12,2 $\pm 21,4$ ( 5 de 0,1 a 240) horas. A duração foi menor que 12, 24 e 36 horas em respectivamente $66,3 \%, 80,5 \%$ e $94,3 \%$ dos indivíduos. Apenas 84 indivíduos informaram a frequência habitual de suas crises. Dentre estes, 25 $(29,7 \%)$ disseram ter crises "raramente", e os demais
A dor foi bilateral em $82,7 \%$ e unilateral em $17,3 \%$ $(n=474$; direita 7,4\%; esquerda 9,9\%). Tiveram dor pulsátil e em aperto 47,5\% e 52,5\% dos entrevistados, respectivamente $(n=480)$. Entre os que tiveram cefaléia, a dor foi considerada leve por $79,3 \%$ e intensa por 20,7\%. Nenhum indivíduo referiu dor insuportável (n=492) (Fig 1c).

A presença de náuseas, vômitos, foto e fonofobia encontra-se demonstrada na Tabela 2. A atividade física rotineira piorou a dor para $41,9 \%$ dos funcionários, melhorou em 7,1\%, e não a alterou em $51,0 \%$.

Cinco e meio por cento dos funcionários preencheram critérios diagnósticos ${ }^{4}$ para enxaqueca. A duração média dos ataques nestes indivíduos foi 13,1 113,5 (8 de 4 a 72) h. A CTT episódica e a CTT crônica estiveram

Tabela 2. Náuseas, vômitos, foto- e fonofobia*.

\begin{tabular}{lccccc}
\hline Sintoma & não & sim & leve & moderado & intenso \\
\hline Náuseas & 83,5 & 16,5 & 7,0 & 3,9 & 5,6 \\
Vômitos & 100 & 0 & 0 & 0 & 0 \\
Fotofobia & 69,6 & 30,4 & 0 & 30,4 & 0 \\
Fonofobia & 68,2 & 31,8 & 0 & 31,8 & 0 \\
\hline
\end{tabular}

*Valores percentuais. 


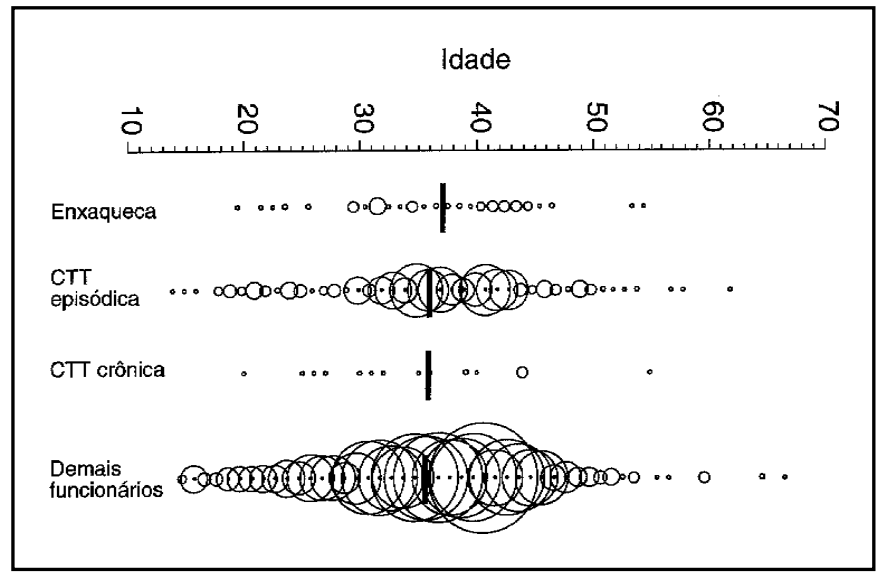

Fig 2. Idade dos funcionários de acordo com o diagnóstico. As barras horizontais representam a média. $O$ diâmetro dos círculos é proporcional ao número de indivíduos com a mesma idade.

presentes em respectivamente $26,4 \%$ e $1,7 \%$ dos entrevistados. A duração média dos ataques na forma episódica foi 13,0 $\pm 21,1$ ( 5 de 2 a 168) h. Indivíduos com a forma crônica tiveram dor por 27,9 $\pm 3,2$ ( 30 de 20 a 30) dias no mês. Em 161 indivíduos (16,2\%) as cefaléias não preencheram critérios nem para enxaqueca (códigos 1,1 ou 1,2 de acordo com a IHS) nem para CTT (códigos 2,1 e 2,2), sendo consideradas como outras cefaléias (OC). A média de idade não variou entre os funcionários com diferentes cefaléias ( $\mathrm{p}=0,7271$ ) (Fig 2).

Quanto ao sexo, as mulheres sofreram significativamente mais cefaléias que os homens $(61,0 \%$ das mulheres; $43,0 \%$ dos homens; $p=0.0001$ ), com maior frequência (mulheres $5,3 \pm 8,0$ ( 2 de 1 a 30 ); homens $3,6 \pm 5,5$ ( 1 de 1 a 30; $\mathrm{p}=0,0081$ ) e intensidade (mulheres $2,0 \pm 0,6$; homens $1,8 \pm 0,7 ; \mathrm{p}=0.0036$ ). A duração dos ataques quase foi diferente estatisticamente (mulheres $13,9 \pm 25,1$ ( 6 de 0,2 a 240 ) h; homens $10,6 \pm 17,5$ ( 5 de 0,1 a $168 \mathrm{~h}$; $\mathrm{p}=0.0897)$. Comparativamente mais mulheres preencheram critérios para enxaqueca $(8,2 \%$ das mulheres contra $3,9 \%$ dos homens; $\mathrm{p}=0,0038)$ e CTT crônica ( $3,2 \%$ das mulheres contra $0,8 \%$ dos homens; $\mathrm{p}=0,0052)$, mas sofreram a mesma quantidade de CTT episódica que os homens (28,9\% das mulheres contra 24,8\% dos homens; $\mathrm{p}=0,1574$ ). As funcionárias tiveram proporcionalmente mais náuseas, foto e fonofobia (Tabela 3), mas suas dores não foram comparativamente mais influenciadas pela atividade física (mulheres: $45,8 \%$; homens: 38,4\%; $\mathrm{p}=0,0972$ ). As cefaléias com intensidade $\geq 2$ (interferem com o trabalho) estavam presentes na mesma proporção nos dois sexos (18,4\% das mulheres e $22,2 \%$ dos homens; $\mathrm{p}=0,3142)$.

Em 102 funcionários $(10,3 \%)$ houve dor com intensidade $\geq 2$. A duração média destes ataques de foi $13,8 \pm 22,7$ ( 6 de 0,5 a 168$) \mathrm{h}$, com frequência de $5,3 \pm 8,0$ ( 2 de 1 a 30 ) ataques por mês. Isto corresponde a uma média de 53,1 $\pm 89,5$ ( 16 de 0,2 a 360) h de cefaléia intensa ( $\geq 2$ ) por mês em cada um destes 102 funcionários.

Tabela 3. Náuseas, foto- e fonofobia em homens e mulheres.

\begin{tabular}{lcccc}
\hline Sintoma & Sexo & $\operatorname{sim}(\%)$ & não $(\%)$ & p (Qui-quadrado) \\
\hline Náuseas & Masculino & 13,0 & 87,0 & $0,0263^{*}$ \\
& Feminino & 20,5 & 79,5 & \\
Fotofobia & Masculino & 23,7 & 76,3 & $0,0019^{*}$ \\
& Feminino & 38,2 & 61,8 & \\
Fonofobia & Masculino & 24,5 & 75,5 & $0,0008^{*}$ \\
& Feminino & 40,6 & 59,4 & \\
\hline
\end{tabular}

*Estatisticamente significativo. 
A Tabela 4 estima os gastos indiretos em função de horas de trabalho com produtividade reduzida. Em relação ao sexo, embora o potencial prejuízo total mensal seja semelhante para homens e mulheres em valores absolutos (R\$5 578,22 e R \$7 535,61, respectivamente, ou US\$4 825.45 e US\$6 518.69), como há mais homens trabalhando, os custos por indivíduo deste ou daquele gênero são 2,1 vezes maiores entre as funcionárias ( $\mathrm{R} \$ 9,07$ US\$7.84 / homem / mês e R\$19,94-US\$17.24 / mulher / mês; p=0,0362).

Tabela 4. Características e custos indiretos das cefaléias.

\begin{tabular}{|c|c|c|c|c|c|}
\hline & $\begin{array}{l}\text { Enxaqueca } \\
\qquad(n=55)\end{array}$ & $\begin{array}{c}\text { CTT } \\
\text { episódica } \\
(\mathrm{n}=262)\end{array}$ & $\begin{array}{l}\text { CTT } \\
\text { crônica } \\
(\mathrm{n}=17)\end{array}$ & $\begin{array}{c}\text { Outra } \\
\text { cefaléia** } \\
(n=161)\end{array}$ & Total \\
\hline $\begin{array}{l}\text { No. funcionários } \\
\text { com dor } \geq 2 \quad(\mathrm{n} / \%)\end{array}$ & $41 / 4,1$ & $24 / 2,4$ & $2 / 0,2$ & $35 / 3,5$ & $102 / 10,3$ \\
\hline $\begin{array}{l}\text { Frequência (crises/mês) } \\
\text { média } \pm \text { desvio padrão } \\
\text { mediana (minimo-máximo) }\end{array}$ & $\begin{array}{c}4,7 \pm 7,4 \\
2(1-30)\end{array}$ & $\begin{array}{c}2,7 \pm 2,9 \\
1(1-12)\end{array}$ & $\begin{array}{l}25,0 \pm 0,0 \\
25(25-25)\end{array}$ & $\begin{array}{c}6,7 \pm 9,5 \\
2(1-30)\end{array}$ & $\begin{array}{l}5,34 \pm 8,0 \\
2(1-30)\end{array}$ \\
\hline $\begin{array}{l}\text { Duração (horas) } \\
\text { média } \pm \text { desvio padrão } \\
\text { mediana (minimo-máximo) }\end{array}$ & $\begin{array}{c}13,0 \pm 12,0 \\
12(4-72)\end{array}$ & $\begin{array}{c}19,8 \pm 34,9 \\
6(2-168)\end{array}$ & $\begin{array}{l}1,25 \pm 1,0 \\
1,2(0,5-2)\end{array}$ & $\begin{array}{c}10,9 \pm 21,9 \\
4(0,2-96)\end{array}$ & $\begin{array}{l}13,8 \pm 22,6 \\
6(0,2-168)\end{array}$ \\
\hline $\begin{array}{l}\text { No. total de horas } \\
\text { com dor } \leq 2 \text { em } 1 \text { mês }\end{array}$ & $2.264,0$ & $1.277,0$ & 62,5 & $1.777,2$ & $5.380,7$ \\
\hline $\begin{array}{l}\text { No. de horas com dor } \\
\text { no trabalho em } 1 \text { mês por } \\
\text { funcionário com dor } \geq 2\end{array}$ & 13,4 & 13,0 & 7,6 & 12,4 & 12,8 \\
\hline $\begin{array}{l}\text { No. de horas com dor } \\
\text { no trabalho em } 1 \text { mês por } \\
\text { funcionário }(n=993)\end{array}$ & 0,5 & 0.3 & 0,01 & 0,4 & 1,3 \\
\hline $\begin{array}{l}\text { Custo por funcionário } \\
\text { por diagnóstico*** } \\
\text { em } 1 \text { mês (R\$) } \\
\text { em } 1 \text { ano (R\$) } \\
\text { em } 1 \text { mês (US\$) } \\
\text { em } 1 \text { ano (US\$) }\end{array}$ & $\begin{array}{c}100,62 \\
1.106,84 \\
87,04 \\
957,47\end{array}$ & $\begin{array}{c}11,91 \\
131,05 \\
10,30 \\
113,36\end{array}$ & $\begin{array}{c}8,98 \\
98,85 \\
7,76 \\
85,51\end{array}$ & $\begin{array}{c}26,98 \\
296,82 \\
23,33 \\
256,76\end{array}$ & $\begin{array}{l}- \\
- \\
- \\
-\end{array}$ \\
\hline $\begin{array}{l}\text { Custo por funcionário (n=993) } \\
\text { em } 1 \text { mês (R\$) } \\
\text { em } 1 \text { ano (R\$) } \\
\text { em } 1 \text { mês (US\$) } \\
\text { em } 1 \text { ano (US\$) }\end{array}$ & $\begin{array}{c}5,57 \\
61,27 \\
4,81 \\
53,00\end{array}$ & $\begin{array}{c}3,14 \\
34,54 \\
2,71 \\
29,87\end{array}$ & $\begin{array}{l}0,15 \\
1,65 \\
0,12 \\
1,42\end{array}$ & $\begin{array}{c}4,38 \\
48,18 \\
3,78 \\
41,67\end{array}$ & $\begin{array}{c}13,24 \\
145,64 \\
11,45 \\
125,98\end{array}$ \\
\hline $\begin{array}{l}\text { Custo total para } \\
993 \text { funcionários }\end{array}$ & & & & & \\
\hline $\begin{array}{l}\text { em } 1 \text { mês (R\$) } \\
\text { em } 1 \text { ano (R\$) } \\
\text { em } 1 \text { mês (US\$) } \\
\text { em } 1 \text { ano (US\$) }\end{array}$ & $\begin{array}{c}5.534,22 \\
60.876,44 \\
4.787,38 \\
52.661,28\end{array}$ & $\begin{array}{c}3.121,56 \\
34.337,11 \\
2.700,31 \\
29.703,90\end{array}$ & $\begin{array}{c}152,78 \\
1.680,56 \\
132,16 \\
1.453,77\end{array}$ & $\begin{array}{c}4.344,39 \\
47.788,28 \\
3.758,12 \\
41.339,34\end{array}$ & $\begin{array}{c}13.152,95 \\
144.682,39 \\
11.377,98 \\
125.157,77\end{array}$ \\
\hline
\end{tabular}

*Valores em Reais e Dólares americanos. Custos avaliados considerando como R $\$ 10,00-$ US $\$ 8,65$ o custo para a empresa da hora trabalhada por funcionário. **Outra cefaléia: Intensidade $\geq 2$, mas sem preencher critérios para enxaqueca ou CTT. ***Total de funcionários por diagnóstico com dor de qualquer intensidade. 


\section{DISCUSSÃO}

Neste estudo, a prevalência das cefaléias foi avaliada em um período retrospectivo de 30 dias. Numa extensa revisão epidemiológica ${ }^{10}, 20$ estudos avaliaram a frequência das crises de enxaqueca, CTT e cefaléia leve / intensa. Na maioria deles os indivíduos sofreram uma ou mais crises por mês (Tabela 5). A média de todos estes estudos (52,6\%) é semelhante ao percentual na nossa população com pelo menos 1 crise no mês (49,8\%). Em um estudo envolvendo 4061 indivíduos, pessoas com enxaqueca e CTT tiveram, em média, respectivamente, 2,82 e 2,89 crises $/ \mathrm{mês}^{11}$. Com períodos de observação mais longos, a incapacidade de recordar adequadamente os fatos relacionados à saúde prejudica a confiabilidade das informações. Embora em 1 mês algumas crises com frequência relativamente baixa possam não ser detectadas, os dados disponíveis sugerem que este período é adequado para avaliar a presença de cefaléias recorrentes na maioria dos indivíduos.

A CTT episódica foi a afecção observada mais frequentemente (26,4\%), seguida da enxaqueca $(5,5 \%)$ e da CTT crônica $(1,7 \%)$. Poucos estudos epidemiológicos foram realizados utilizando os critérios da IHS. Pelo estudo de Göbel et al., a prevalência ao longo da vida é 27,5\% para a enxaqueca, $38,3 \%$ para a CTT episódica e 3\% para a CTT crônica ${ }^{11}$. Na Dinamarca, a mesma prevalência foi estimada em 9\% (enxaqueca sem aura), 6\% (enxaqueca com aura), 66\% (CTT episódica) e 3\% (CTT crônica) ${ }^{5}$. Considerando que no presente estudo a prevalência em 30 dias foi utilizada, valores ligeiramente mais baixos foram obtidos. A proporção entre os diagnósticos no presente estudo é compatível com os dados epidemiológicos disponíveis.

Tabela 5. Estudos quanto à freqüência dos ataques de cefaléia.

\begin{tabular}{|c|c|c|}
\hline Autor (ano) & $\begin{array}{c}\text { Afecção estudada } \\
\text { (período de observação) }\end{array}$ & $\begin{array}{c}\text { Indivíduos com } \\
\geq 1 \text { crise/mês }(\%)\end{array}$ \\
\hline Ogden, 1952 & Cefaléia & 48,0 \\
\hline Clarke \& Waters, 1974 & Cefaléia (último ano) & 52,0 \\
\hline Mills \& Waters, 1974 & Cefaléia leve (último ano) & 36,0 \\
\hline Mills \& Waters, 1974 & Cefaléia intensa (último ano) & 32,1 \\
\hline Moss \& Waters, 1974 & Cefaléia leve (último ano) & 50,2 \\
\hline Moss \& Waters, 1974 & Cefaléia intensa (último ano) & 19,4 \\
\hline Quinnell \& Waters, 1974 & Cefaléia leve (último ano) & 67,0 \\
\hline Quinnell \& Waters, 1974 & Cefaléia intensa (último ano) & 64,5 \\
\hline Small \& Waters, 1974 & Cefaléia leve (último ano) & 40,5 \\
\hline Small \& Waters, 1974 & Cefaléia intensa (último ano) & 27,3 \\
\hline Sillanpää, 1976 & Cefaléia (último ano) & 23,5 \\
\hline Ekbom et al, 1978 & Enxaqueca & 85,2 \\
\hline Ekbom et al, 1978 & Cefaléia não enxaquecosa & 91,1 \\
\hline Nikiforow \& Hokkanen, 1978 & Cefaléia leve (último ano) & 84,2 \\
\hline Nikiforow \& Hokkanen, 1978 & Cefaléia intensa (último ano) & 55,7 \\
\hline Sparks, 1978 & Enxaqueca & 43,0 \\
\hline Andrassik et al., 1979 & Cefaléia (último ano) & 83,7 \\
\hline Sillanpää, 1983 & Cefaléia (último ano) & 28,5 \\
\hline Passchier \& Orlebeke, 1985 & Cefaléia (último ano) & 44,4 \\
\hline Linet et al., 1989 & Cefaléia (último mês) & 67,4 \\
\hline Phanthumchinda \& Sithi-Amorn, 1989 & Enxaqueca (último ano) & 97,4 \\
\hline Henry et al., $1992 *$ & Enxaqueca & 82,0 \\
\hline Pryse-Phillips et al., 1992* & Enxaqueca (último ano) & 34,0 \\
\hline Pryse-Phillips et al., 1992* & CTT (último ano) & 31,0 \\
\hline Rasmussen et al., 1992* & Enxaqueca (último ano) & 22,9 \\
\hline \multirow[t]{2}{*}{ Stewart, $1992 *$} & Enxaqueca (último ano) & 57,0 \\
\hline & Média: & 52,6 \\
\hline
\end{tabular}

Extraído e modificado a partir de Stewart et al., $1994^{10}$. *Diagnóstico segundo a IHS. 
Os 993 indivíduos estudados foram entrevistados em seu ambiente de trabalho, o que os tornam mais representativos da população empregada em geral. Pacientes com cefaléias menos intensas e menor impacto individual (justamente as mais frequentes), tendem a negligenciar sua dor. Segundo Rasmussen, $44 \%$ das pessoas com cefaléia não visitam qualquer médico ${ }^{5}$. Apenas $16 \%$ dos pacientes com CTT procuram atendimento, contra 56\% dos enxaquecosos (procuram especialista: $16 \%$ na enxaqueca e $4 \%$ na CTT) ${ }^{12}$. Nos EUA, $2 / 3$ dos enxaquecosos nunca procuraram um médico ${ }^{13}$. No Canadá, apenas $54 \%$ dos enxaquecosos e $45 \%$ dos pacientes com CTT já haviam procurado atendimento médico, e apenas $32 \%$ retornaram para acompanhamento ${ }^{14}$. Assim, levantamentos realizados em instituições de saúde podem mostrar prevalência relativamente maior de enxaquecosos em relação à CTT, o que não corresponde à realidade na população geral. O presente estudo confirma estas observações e ilustra como cefaléias podem ser potencialmente mais frequentes do que a proporção de indivíduos que procura atendimento.

Mulheres sentem mais dores recorrentes que homens ${ }^{15}$, incluindo a maioria das cefaléias ${ }^{16}$. Este estudo mostra que mulheres têm mais enxaquecas e mais estigmas enxaquecosos que os homens. Embora ambos os sexos tenham cefaléia $\geq 2$ em proporções semelhantes, a intensidade, a frequência e o custo (por indivíduo do mesmo sexo) das crises foram maiores nas mulheres. $\mathrm{O}$ sexo feminino predomina na enxaqueca (razão 1:2 a 1:3) e na CTT (razão em torno de 1:1,5) ${ }^{16-18}$. Entre enxaquecosos nos EUA, mais da metade das mulheres e mais de 1/3 dos homens perdem mais de 6 dias de trabalho por ano ${ }^{13}$.

As cefaléias primárias acometem preferencialmente adultos jovens, sobretudo na faixa etária mais produtiva para o trabalho ${ }^{2,5,19}$. No que se refere à idade, a população estudada não representa adequadamente a da população geral, pois os idosos ou os muito jovens não fazem parte da força de trabalho. Assim , a prevalência deve estar acima da esperada para a população geral, mas representa mais especificamente o acometimento em um grupo de trabalhadores.

O impacto causado pelas cefaléias, principalmente a enxaqueca, está longe de ser desprezível ${ }^{6-}$ 9,13,19,20. Isto se deve à frequência relativamente elevada e ao caráter crônico. Em um levantamento telefônico realizado no Canadá entre 1573 adultos, 59\% tinham pelo menos um membro na residência com cefaléia. Metade dos enxaquecosos interrompiam sua atividade devido à dor e $1 / 3$ necessitavam de repouso, correspondendo, no Canadá, a cerca de 7 milhões de dias de trabalho perdidos por ano ${ }^{21}$. Dezenove a $43 \%$ dos enxaquecosos perdem pelo menos um dia de trabalho por ano, e 1/3 precisam cancelar compromissos pessoais ou sociais ${ }^{14}$. $\mathrm{Na}$ Inglaterra, entre 476 funcionários de um centro de pesquisa e comércio na área de indústria química (perfil semelhante à população deste estudo), $27,4 \%$ dos enxaquecosos disseram que a dor interferia com a sua produtividade pelo menos moderadamente, e $63 \%$ consideraram que havia alguma interferência, mas menos importante ${ }^{22}$. Nos EUA, um questionário aplicado em 13343 indivíduos, 7,7\% perderam em um ano pelo menos um dia inteiro de trabalho devido a cefaléias (60\% delas enxaquecas, $13 \%$ eram CTT, e $27 \%$ outro tipo). Dentre os que sofreram cefaléias no último ano, $31 \%$ admitiram que sua produtividade era afetada mais do que raramente ${ }^{23}$. Entre 740 indivíduos estudados na Dinamarca, $43 \%$ dos enxaquecosos e $12 \%$ dos indivíduos com CTT perderam pelo menos um dia inteiro de trabalho durante o ano (mais comum: 1-7 dias). Para cada 1000 habitantes a enxaqueca tende a produzir 270 dias perdidos de trabalho por ano ${ }^{12}$. Cerca de $10 \%$ dos funcionários, no presente estudo disseram que suas crises prejudicam o trabalho. De acordo com a freqüência e duração, rstima-se que 538,75 horas foram pagas e não plenamente trabalhadas durante 1 mês. Na mesma proporção, uma empresa com 10000 empregados perderia $5425,48 \mathrm{~h}$, ou 7,5 meses (5,3 h/mês/funcionário da empresa). O número de horas pagas e não trabalhadas devido a cefaléias em um mês corresponderá a um prejuízo anual de 144 mil reais, apenas entre os 993 indivíduos entrevistados. Cada funcionário pode custar à empresa até cerca de $\mathrm{R} \$ 130,00$-US $\$ 112.45$ por mês. Transpondo estes valores para toda a Petrobrás (41 000 funcionários), a ocorrência de cefaléias pode ocasionar um prejuízo anual da ordem de R\$5 971 240,00-US\$5 165432.52. 
O prejuízo real, entretanto, pode ser maior devido aos seguintes fatores: 1) Os indivíduos tendem a esquecer ou minimizar a importância de suas crises, sobretudo se ocorreram há mais de 2 semanas; 2) Existe a possibilidade de indivíduos omitirem ou minimizarem a interferência no seu trabalho temendo represálias por parte da empresa; 3) Funcionários podem ter proporcionalmente mais horas com cefaléia durante o período de trabalho devido a estresse ou cansaço. Além disto, há menos cefaléias durante o sono, e no presente estudo consideramos uma probabilidade igual para ocorrência de cefaléia ao longo das 24 horas; 4) Estudos de prevalência por períodos mais prolongados podem demonstrar a presença de mais crises;

Por outro lado, a estimativa poderia ser exagerada, pois: 1) A interferência no trabalho pode ser apenas parcial. Assim, o funcionário poderia trabalhar com parte da sua produtividade intacta. Nós consideramos que o número de horas com dor intensa impediu o rendimento; 2) $\mathrm{O}$ custo de R $\$ 10,00-\mathrm{US} \$ 8.65$ / hora pode ser menor em trabalhos menos qualificados.

A estimativa do custo de uma doença como a enxaqueca pode empregar diferentes metodologias ${ }^{10}$. Enxaquecosos tendem a perder até 6,7 dias de trabalho por ano ${ }^{10}$, mas a avaliação baseada no absenteísmo pode ser falha, pois os pacientes podem comparecer ao trabalho porém com prejuízo na produtividade ${ }^{24}$. No presente estudo, os custos indiretos foram estimados segundo o preço da hora trabalhada, considerando-se a possibilidade de interferência no trabalho. Na Inglaterra, a enxaqueca pode provocar prejuízos da ordem de US $\$ 1,1$ bilhão ${ }^{25}$. Outro estudo inglês estimou que a perda determinada por 378 enxaquecosos (158 pela IHS e 220 pelo clínico geral) entre 1903 entrevistados é da ordem de $£ 113364.00$, ou cerca de $\mathrm{R} \$ 499,84-\mathrm{US} \$ 432.38$ por enxaquecoso por ano. Os autores consideram que este número pode estar abaixo da realidade ${ }^{26}$. De acordo com a perda na produtividade, o custo anual calculado em 6 milhões de enxaquecosos empregados nos EUA é US $\$ 1,4$ bilhões (US\$226 por enxaquecoso empregado). Lipton e von Korff consideram que estes valores certamente são maiores, pois haveria erros metodológicos no seu cálculo ${ }^{13}$. Se considerarmos a estimativa máxima para os EUA (US\$17 bilhões/ano), encontraríamos algo em torno de US\$2 744.00/ano por enxaquecoso empregado. Cada indivíduo com enxaqueca no presente estudo custa US $\$ 914.00$ por ano, o que está dentro das estimativas americanas. Entretanto, deve-se considerar as diferenças metodológicas. Para avaliação dos custos anuais, nós utilizamos como base crises que interferiram com o trabalho durante 1 mês, supondo que também o fariam ao longo de todo o ano. Porém, a mesma afecção pode interferir com o trabalho apenas em parte das crises, ou reduzir a produtividade parcialmente. Assim, o prejuízo seria menor. Não obstante, é metodologicamente difícil determinar com precisão o momento a partir do qual uma dor que se torna mais intensa passa a interferir com o trabalho. Para fins práticos, os dados obtidos neste estudo devem ser encarados como o potencial prejuízo sofrido pela empresa caso os funcionários tenham as mesmas dores nos outros meses do ano.

Em relação aos custos de acordo com os diferentes diagnósticos (Tabela 4), a enxaqueca, a cefaléia que determina o maior prejuízo, acomete pessoas por tempo mais curto que a CTT episódica, porém custa mais. Por outro lado, a CTT crônica é a que mais longamente ataca os pacientes, pois necessariamente ocorre em mais de 15 dias por mês. Entretanto, como a intensidade é baixa, o grau de interferência no trabalho é significativamente menor. As OC são quase tão custosas quanto a enxaqueca (Tabela 4). Este grupo envolve pacientes eventualmente classificados pela IHS como 1,7 (desordem enxaquecosa que não preenche os critérios) ${ }^{4}$. Em uma empresa que visa reduzir custos indiretos determinados por cefaléias, a enxaqueca deve receber proporcionalmente maior atenção.

A conclusão mais importante deste estudo é que cefaléias são frequentes e podem custar caro às empresas também no Brasil. Não obstante, seu tratamento é comparativamente barato e o índice de sucesso, compensador. Pacientes com cefaléias primárias não requerem equipamentos ou exames complementares para o diagnóstico, o que torna seu manejo menos oneroso. Investir na identificação e no tratamento apropriado das cefaléias constitui, portanto, importante medida de redução de custos. 
A profilaxia contra cefaléias recidivantes é satisfatoriamente eficaz, pelo menos $40 \%$ mais eficiente que placebo ${ }^{27}$. Beta-bloqueadores, por exemplo, reduzem em mais de $50 \%$ a frequência dos ataques em até $80 \%$ dos pacientes ${ }^{28}$. Como o custo dos medicamentos profiláticos é relativamente baixo, o investimento na identificação, diagnóstico e tratamento adequado é necessário e, comparativamente, compensador ${ }^{25}$. Em um estudo realizado na Suécia com 99 enxaquecosos tratados durante 6 meses, verificou-se significativa melhora na qualidade de vida relacionada à saúde, diminuição do número de crises e do absenteísmo ${ }^{29}$.

O sumatriptan, o primeiro dentre uma série de medicamentos anti-enxaquecosos agonistas 5$\mathrm{HT}_{1 \mathrm{D}}{ }^{30-32}$, elimina em até $87 \%$ dos casos a crise de enxaqueca em 2 horas $^{31}$. Novas drogas desta família, mais eficientes e com menos efeitos colaterais, poderão em breve contribuir para melhorar estes índices. Mesmo o uso do sumatriptan, um medicamento comparativamente mais caro, mostrase compensador, tendo em vista a redução dos custos indiretos ${ }^{33}$. Em um levantamento entre 220 indivíduos que usaram sumatriptan injetável, cerca de $2 / 3$ eram empregados em tempo integral. Nestes, houve uma redução de $71 \%$ na falta ao trabalho com o uso do sumatriptan, e uma diminuição de $20 \%$ dos dias de trabalho com sintomas. A produtividade aumentou $85 \%$. Cada empregado enxaquecoso foi associado a um custo mensal de US\$681.00 (sem sumatriptan) e US\$246.00 (com sumatriptan), o que representa uma economia US\$435.00 por indivíduo. Como o custo do remédio foi de US $\$ 43,78$, o benefício foi cerca de 10 vezes maior que o custo $^{34}$. Sumatriptan também mostrouse mais vantajoso economicamente quando comparado à cafeína / ergotamina ${ }^{35}$. A combinação profilaxia adequada + tratamento agudo tende a reduzir significativamente os custos indiretos determinados pelas cefaléias ${ }^{25}$.

Além de melhorar a saúde de seus funcionários, o que tem repercussões positivas na sua imagem, a empresa que investe no combate às cefaléias primárias ganha potencialmente mais do que a simples redução dos custos diretamente relacionados às crises. A eliminação da ansiedade $\mathrm{e}$ outras desordens afetivas relacionadas à dor crônica melhora a qualidade de vida do funcionário e tende a aumentar ainda mais a sua produtividade. Muitos enxaquecosos vivem temendo o próximo ataque, o que Blau descreve como "melontofobia"36. Os pacientes enxaquecosos têm qualidade de vida subjetiva e sensação de bem-estar menores, mesmo entre as crises, quando comparados a controles $^{37}$. A profilaxia tende a aumentar o bem-estar, eliminado os sintomas inter-crises.

Para que os custos, porém, sejam reduzidos satisfatoriamente, é necessário:

1) Entrevistar a população alvo e identificar os indivíduos que necessitam de tratamento. Investir apenas na disponibilidade de consultas no setor de medicina do trabalho não traz benefícios aos funcionários, pois a demanda espontânea é pequena e o médico não é treinado para atendimento em cefaléias. Questionários auto-aplicados são pouco eficientes na identificação dos pacientes sujeitos a tratamento ${ }^{38}$.

2) Submeter os indivíduos selecionados a acompanhamento especializado, monitorando-os por diário padronizado ${ }^{39}$. A maioria dos pacientes com cefaléias crônicas são mal examinados, maldiagnosticados e tratados inadequadamente. Em um levantamento realizado nos EUA, entre 2479 enxaquecosos apenas $38 \%$ foram diagnosticados por um médico ${ }^{40}$. Existem diretrizes para a constituição do atendimento ideal em cefaléias, raramente respeitadas ${ }^{41}$.

Enquanto a cura definitiva das cefaléias primárias ainda não está disponível, medidas terapêuticas simples e baratas podem reduzir significativamente o impacto individual e social destas afecções. As evidências disponíveis indicam que, além do bem-estar individual, o tratamento adequado das cefaléias recidivantes pode produzir benefícios significativos para a coletividade, incluindo trabalhadores de uma empresa.

Agradecimentos - Os autores agradecem os valiosos comentários do Dr. João José Freitas de Carvalho e Prof. Dr. Ricardo Gattass. Agradecemos ainda a todos os funcionários do CENPES e ao Prof. Dr. Armando Pimenta, do Serviço de Saúde da empresa. 


\section{REFERÊNCIAS}

1. Stewart WF, Shechter BA, Rasmussen BK. Migraine prevalence: a review of population-based studies. Neurology 1994;44(Suppl 4):17-23

2. Lipton RB, Stewart WF. Epidemiology of migraine and other primary headache disorders. In Gorelick PB, Alter M (eds). Handbook of neuroepidemiology. New York: Marcel Dekker, 1994;357-379

3. Linet MS, Stewart WF, Celentano DD. An epidemiologic study of headache among adolescents and young adults. JAMA 1989;261:2211-2216.

4. Headache Classification Committee of the International Headache Society. Classification and diagnostic criteria for headache disorders, cranial neuralgias and facial pain. Cephalalgia 1988;8(Suppl 7):1-96

5. Rasmussen BK. Epidemiology of headache. Cephalalgia 1995;15:45-68.

6. Lipton RB, Stewart WF, Von Korff M. The Burden of Migraine. Pharmacoeconomics 1994;6:215-221.

7. de Lissovoy G, Lazarus SS. The economic cost of migraine: present state of knowledge. Neurology 1994;44(Suppl 4):56-62.

8. Osterhaus JT, Gutterman DL, Plachetka JR. Healthcare resources and lost labour costs of migraine in the US. Pharmacoeconomics 1992;2:67-76.

9. Stang PE, Osterhaus JT. Impact of migraine in the United States: data from the national health interview survey. Headache 1993;33:29-35.

10. Stewart WF, Shechter A, Lipton RB. Migraine heterogeneity: disability, pain intensity, and attack frequency and duration. Neurology 1994;44(Suppl 4):24-39.

11. Göbel H, Petersen-Braun M, Soyka D. The epidemiology of headache in Germany: a nationwide survey of a representative sample on the basis of the headache classification of the International Headache Society. Cephalalgia 1994;14:97-106.

12. Rasmussen BK, Jensen R, Olesen J. Impact of headache on sickness absence and utilisation of medical services: a Danish population study. J Epidemiol Community Health 1992;46:443-446.

13. Lipton RB, Von Korff M. Migraine impact and funcional disability. Cephalalgia 1995;15:4-9.

14. Edmeads J, Findlay H, Tugwell P, Pryse-Phillips W, Nelson RF, Murray TJ. Impact of migraine and tension-type headaches on lifestyle, consulting behaviour, and medication use: a Canadian population survey. Can J Neurol Sci 1993;20:131-137.

15. Unruh AM. Gender variations in clinical experience. Pain 1996;65:123-167.

16. Rasmussen BK, Olesen J. Epidemiology of migraine and tension-type headache. Curr Opin Neurol 1994;7:264-271.

17. Lipton RB, Silberstein SD, Sewart WF. An update on the epidemiology of migraine. Headache 1994;34:319-328.

18. Henry P, Michel P, Brochet B, Dartiguea JF, Tiaon S, Salamon R. A nationwide survey of migraine in France: prevalence and clinical features in adults. Cephalalgia 1992;12:229-237.

19. Lipton RB, Stewart WF, Von Korff M. Burden of migraine: societal costs and therapeutic opportunities. Neurology 1997;48(Suppl 3):S4-S9.

20. Stewart WF, Lipton RB. The economic and social impact of migraine. Eur Neurol 1994;34(Suppl 2):12-17.

21. Pryse-Phillips W, Findlay H, Tugwell P, Edmeads J, Murray TJ, Nelson RF. A Canadian population survey on the clinical, epidemiologic and societal impact of migraine and tension-type headache. Can J Neurol Sci 1992;19:333-339.

22. Mounstephen AH, Harrison RK. A study of migraine and its effects in a working population. Occup Med 1995;45:311-317.

23. Schwartz BS, Stewart WF, Lipton RB. Lost workdays and decreased work effectiveness associated with headache in the workplace. JOEM 1997;34:320-327.

24. Celentano DD, Stewart WF, Lipton RB, Reed ML. Medication use and disability among migraineurs: a national probability sample survey. Headache 1992;32:223-228.

25. Cull RE, Wells NEJ, Miocevich ML. The economic cost of migraine. Br J Med Econ 1992;2:103-115.

26. Clarke C, Macmillan L, Sondhi S, Wells N. Economic and social impact of migraine. J Assoc Physicians India 1996;89:77-84.

27. Tfelt-Hansen P. Prophylactic treatment of migraine: evaluation of clinical trials and choice among drugs. Cephalalgia 1995;15(Suppl 15):29-32.

28. Silberstein SD, Lipton RB. Overview of diagnosis and treatment of migraine. Neurology 1994;44(Suppl 7):S6-S16.

29. Dahlöf CGH. Health-related quality of life under six months' treatment of migraine: an open clinic-based longitudinal study. Cephalalgia 1995;15:414-422.

30. Peroukta SJ. Sumatriptan in acute migraine: pharmacology and review of world experience. Headache 1990;30(Suppl 2):554-560.

31. Tfelt-Hansen P. Sumatriptan for the treatment of migraine attacks: a review of controlled trials. Cephalalgia 1993;13:238-244.

32. Ferrari MD, Saxena PR. Clinical and experimental effects of sumatriptan in humans. Trends Pharmacol Sci 1993;14:129-133.

33. Mushet GR, Miller D, Clements B, Pait G, Gutterman DL. Impact of sumatriptan on workplace productivity, nonwork activities, and health-related quality of life among hospital employees with migraine. Headache 1996;36:137-143.

34. Legg RF, Sclar DA, Nemec NL, Tarnai J, Mackowiak JI. Cost benefit of sumatriptan to an employer. JOEM 1997;39:652-657.

35. Evans KW, Boan JA, Evans JL, Shuaib A. Economic evaluation of oral sumatriptan compared with oral caffeine/ergotamine for migraine. Pharmacoeconomics 1997;12:565-577.

36. Blau JN. A plain man's guide to the management of migraine. Br Med J 1982;284:1095-1097.

37. Dahlöf CGH, Dimenäs E. Migraine patients experience poorer subjective well-being/quality of life even between attacks. Cephalalgia 1995;15:31-36.

38. Rasmussen BK, Jensen R, Olesen J. Questionnaire versus clinical interview in the diagnosis of headache. Headache 1991;31:290-295.

39. Russell MB, Rasmussen BK, Brennum J, Iversen HK, Jensen R, Olesen J. Presentation of a new instrument: the diagnostic headache diary. Cephalalgia 1992;12:369-374.

40. Lipton RB, Stewart WF, Celentano DD, Reed ML. Undiagnosed migraine headaches. A comparison of symptom-based and reported physician diagnosis. Arch Intern Med 1992;152:1273-1278.

41. Jensen K, Campbell K, Diener HC, Kurdi A, Mathew N, Sakai F, Vincent M, Vein AM, (Task force of the International Headache Society). Organization and delivery of services to headache patients. Cephalalgia 1997;17:702-710. 